\title{
Downregulation of human Wnt3 in gastric cancer suppresses cell proliferation and induces apoptosis
}

\author{
This article was published in the following Dove Press journal: \\ OncoTargets and Therapy \\ 27 June 2016 \\ Number of times this article has been viewed
}

\author{
Hai-Sheng Wang, ${ }^{1} *$ Xiaobo \\ Nie, ${ }^{2, *}$ Rui-Bing Wu,' Hong- \\ Wei Yuan,' Yue-Hong Ma,' \\ Xiu-Lan Liu,' Jian-Yu Zhang,' \\ Xiu-Ling Deng,' Qin Na,' \\ Hai-Yan Jin,' Yan-Chao Bian,' \\ Yu-Min Gao, ${ }^{3}$ Yan-Dong \\ Wang, ${ }^{4}$ Wei-Dong Chen, ${ }^{1,2}$ \\ 'Key Laboratory of Molecular Pathology, \\ School of Basic Medical Science, Inner \\ Mongolia Medical University, Hohhot, \\ ${ }^{2}$ Key Laboratory of Receptors-Mediated \\ Gene Regulation and Drug Discovery, \\ School of Medicine, Henan University, \\ Kaifeng, ${ }^{3}$ Epidemiology Section, \\ Public Health School, Inner Mongolia \\ Medical University, Hohhot, ${ }^{4}$ State Key \\ Laboratory of Chemical Resource \\ Engineering, College of Life Science \\ and Technology, Beijing University of \\ Chemical Technology, Beijing, People's \\ Republic of China \\ *These authors contributed equally \\ to this work
}

\begin{abstract}
Aberrant activation of Wnt/ $\beta$-catenin signaling pathways is closely involved in the occurrence and progression of several types of human malignancies. However, as a fundamental component in this cascade, Wnt3 has not been well understood for the expression level and pathogenic mechanism in gastric carcinogenesis. Here, this research was undertaken to elucidate the important role of Wnt3 in gastric cancer. Wnt3 expression in gastric carcinomas and their respective normal tissues was examined by immunoblotting and immunohistochemistry. In all cases, Wnt3 expression was significantly elevated in gastric carcinomas compared with normal tissues. Knocking down Wnt3 in MGC-803 gastric cancer cells by small interfering RNAs transfection led to an obvious decrease in both transcript and protein levels. Silence of Wnt3 expression in gastric cancer cells inhibited the expression of $\beta$-catenin and cyclin D1 genes in Wnt/ $\beta$-catenin pathway, significantly blocked cellular proliferation, delayed cell cycle, suppressed cell invasion and metastasis, accompanied by a higher apoptosis rate. Together, we conclude that upregulation of Wnt3 plays a crucial role in gastric tumorigenesis by inducing proliferation, migration, and invasion and inhibiting apoptosis of cancer cells, and Wnt3 might be a potential target for the treatment of gastric cancer.
\end{abstract}

Keywords: gastric carcinogenesis, gastric carcinoma, $\beta$-catenin, siRNA, invasion

\section{Introduction}

Gastric cancer is the fifth most common cancer and the third leading cause of cancer-related death in worldwide, with more than 900,000 new cases diagnosed annually. ${ }^{1}$ It is a complex polygenetic disease ascribed to the interactions between genetic predisposition and environmental factors. Helicobacter pylori infection is the frequent risk factor in more than $65 \%$ of gastric cancers. ${ }^{2}$ In early stages, gastric cancer is curable through gastrectomy combined with radiation therapy and chemotherapy and the cure rate exceeds $90 \%$. The routine use of gastroscopic examination and computed tomography scanning has significantly improved the detection of gastric cancer and living standard, and effective strategies on the eradication of $H$. pylori have significantly reduced its incidence in the past few decades. Unfortunately, gastric cancer often reaches to an advanced stage or metastasizes because of no noticeable or unspecific symptom in early stage. Therefore, the prognosis of gastric cancer still remains poor and the 5-year survival rate is reported to be less than $10 \%{ }^{3}$ Despite the fact that great research efforts have been taken in this field, it is still meaningful to explore novel molecular markers and detailed molecular mechanisms contributing to improving diagnostic and therapeutic management of gastric cancer.

Named for Drosophila wingless and mouse homolog Int-1 (Wnt1), ${ }^{4}$ Wnt gene family encodes a group of glycoproteins which are evolutionarily conserved across 
metazoans, and functions in a paracrine or autocrine mode to modulate embryonic development, regeneration of adult tissues, and many other processes in all animal species. ${ }^{5}$ In humans, Wnt/ $\beta$-catenin signaling pathways are activated upon binding of 19 known Wnt proteins to the corresponding member of frizzled family and co-receptor LRP5/6, resulting in the recruitment of disheveled and destruction of protein complex, the ensuing events prevent degradation of $\beta$-catenin and facilitate its accumulation in nucleus, thus to activate transcription of several genes controlling diverse biological processes. In contrast, $\beta$-catenin is degraded by proteasome in the absence of Wnt ligands. ${ }^{6}$ On the other hand, it has been proved that mutations or deregulated expression of components of Wnt pathways play critical role in the development of multiple human malignancies. ${ }^{7}$ Therefore, extensive research efforts have been driven at targeting Wnt pathway in cancer drug discovery, albeit many factors have impeded progress in this field, such as abundant number of components involved in Wnt signaling cascade and complex cross talk with developmental processes. ${ }^{8}$

As a member of Wnt family, Wnt3 was reported to be relevant in hepatic, lung, and colorectal carcinogenesis. ${ }^{9-11}$ In this study, we showed that the expression of Wnt3 was upregulated in gastric carcinomas compared with the normal tissues. Furthermore, we identified that knocking down Wnt3 using small interfering RNA (siRNA) downregulated the gene expression of $\beta$-catenin and cyclin D1, halted cell cycle, suppressed the proliferation, invasion, and metastasis, but promoted cellular apoptosis in gastric cancer cells. Our findings indicate that Wnt3 could contribute to gastric carcinogenesis by promoting cellular proliferation and metastasis and may be a potential therapeutic target for gastric cancer.

\section{Materials and methods Immunohistochemistry}

The paraffin-embedded gastric cancer tissues were collected from Pathology Department of Huaihe Hospital affiliated to Henan University (Kaifeng, People's Republic of China). The immunohistochemistry was performed using the following protocol. Briefly, the tissues were incubated at $65^{\circ} \mathrm{C}$ for 1 hour and rehydrated through an ethanol gradient. The section was boiled in $1 \mathrm{mM}$ ethylenediaminetetraacetic acid buffer ( $\mathrm{pH} 7.5$ ) for 30 minutes for antigen retrieval, and quenched by immersing in 3\% hydrogen peroxide. After blocking the nonspecific binding with 5\% sheep serum albumin for 30 minutes, the section was incubated with a rabbit antiWnt3 antibody (Abcam, Cambridge, UK) (1:1,500 dilution in $5 \%$ bovine serum albumin in distilled water) overnight at $4^{\circ} \mathrm{C}$, then rinsed three times with phosphate-buffered saline (PBS) and incubated for 30 minutes at $37^{\circ} \mathrm{C}$ with a drop of secondary antibody. After washed with PBS for three times, the section was visualized with 3'3-diaminobenzidine tetrahydrochloride, counterstained with hematoxylin. All sections were observed under microscope and images were randomly taken with the same exposure time and light intensity.

\section{Collection of gastric tissue specimens}

The study was approved by the Ethics Committee of Huaihe Hospital and School of Medicine of Henan University (registration number of the ethics committee: HUSOM2015015), Kaifeng, and written informed consent was obtained from all patients. Human gastric samples (20 cases) were collected in the Department of General Surgery during surgery on patients with gastric cancer, who underwent complete surgical resection for the disease at Huaihe Hospital between May 2015 and August 2015. The patients were selected based on pathological diagnosis of gastric cancer. There was no evidence of any other malignancies and no history of preoperative anticancer treatment. After each surgical removal, the tissue sample was immediately snap-frozen in liquid nitrogen and stored at $-120^{\circ} \mathrm{C}$ until subsequent analysis.

\section{Cell culture}

The MGC-803 and SGC-7901 cell lines were purchased from School of Basic Medicine of Peking Union Medical College (Beijing, People's Republic of China). Cells were cultured in Dulbecco's Modified Eagle's Medium: Nutrient Mixture F-12 (Thermo Fisher Scientific, Waltham, MA, USA) supplemented with 10\% fetal bovine serum (FBS) (Thermo Fisher Scientific) and antibiotics (100 U/mL penicillin and streptomycin) (Thermo Fisher Scientific) at $37^{\circ} \mathrm{C}$ in a humidified atmosphere of $5 \% \mathrm{CO}_{2}$.

\section{Wnt3 knockdown by siRNA transfection in gastric cancer cells}

Four recombined pGPU6 plasmids that express siRNAs targeting Wnt3 messenger RNA (mRNA) were constructed (GenePharma, Shanghai, People's Republic of China) and amplified. The anti-Wnt3-siRNAs targeted sequences at positions 337, 714, 981, or 1188 on the Wnt3 transcript. MGC-803 and SGC-7901 cells were seeded into a six-well plate at a density of $2 \times 10^{5}$ cells per well 16 hours prior to transfection. Cells were transiently transfected with $2.5 \mu \mathrm{g}$ of each siRNA or control siRNA (siRNA-NC) using lipofectamine 2000 (Thermo Fisher Scientific) in serum-free and antibiotic-free medium. The medium was subsequently aspirated and changed with fresh culture medium after 6 hours. 
After 24 or 48 hours, the transfection efficiency was evaluated by recording images of cells at $\times 100$ magnification from the same visual field under the natural light and fluorescence conditions. Subsequently, cells were harvested and extracted for further analysis.

\section{RNA extraction and quantitative real- time polymerase chain reaction}

Total RNA was extracted from cells after transfection using an RNAsimple Total RNA kit (Tiangen, Beijing, People's Republic of China) according to the manufacturer's instructions. The RNA was quantified using a nanodrop reader and $2 \mu \mathrm{g}$ of RNA was reverse transcribed to cDNA using the Strand cDNA Synthesis kit (Thermo Fisher Scientific). Briefly, total RNA was mixed with $1 \mu \mathrm{L}$ random hexamer primer, made up to a $12 \mu \mathrm{L}$ mixture with nuclease-free water, and heated at $65^{\circ} \mathrm{C}$ for 5 minutes, followed by chilled on ice. After primer hybridization, $8 \mu \mathrm{L}$ reaction volume containing $5 \times$ reaction buffer, RiboLock RNase inhibitor (20 U/ $\mu \mathrm{L}), 10 \mathrm{mM}$ deoxynucleotide mix, and RevertAid M-MuLV reverse transcriptase $(200 \mathrm{U} / \mu \mathrm{L})$ were added to the RNA and incubated for 5 minutes at $50^{\circ} \mathrm{C}$ followed by 60 minutes at $42^{\circ} \mathrm{C}$. The quantitative realtime polymerase chain reaction analyses were performed using $200 \mathrm{ng}$ cDNA per reaction with SYBR green assay with primer set designed to amplify a $157 \mathrm{bp}$ fragment of Wnt3 (forward, 5'-CCACAACACGAGGACGGAGA-3'; reverse, 5'-CGCCCAGCCACACACTTC-3'), a 183 bp fragment of $\beta$-catenin (forward, 5'-GCGCCATTTTAAGCCTCTCG-3'; reverse, 5'-AAATACCCTCAGGGGAACAGG-3'), a 232 bp fragment of FZD7 (forward, 5'-CGCTCATGAAC AAGTTCGGC-3'; reverse, 5'-CATGAGAAGGGGAA GGCGG-3'), a 216 bp fragment of cyclin D1 (forward, 5'-AGTTGCAAAGTCCTGGAGCC-3'; reverse, 5'-GTTTCCACTTCGCAGCACAG-3') or using a primer pair complementary to the human glyceraldehyde 3-phosphate dehydrogenase cDNA (forward, 5'-CCATCACCATCTTCCAGGAGCG-3'; reverse, 5'-AGAGATGATGACCCTTTTGGC-3') with the following conditions: denaturation at $95^{\circ} \mathrm{C}$ for 3 minutes followed by 40 cycles of denaturation at $95^{\circ} \mathrm{C}$ for 30 seconds and annealing and extension at $62^{\circ} \mathrm{C}$ for 40 seconds. Melting curve analysis $\left(65^{\circ} \mathrm{C}-95^{\circ} \mathrm{C}\right)$ was routinely performed to confirm the specificity of primers at the end of the assay.

\section{Protein extraction and immunoblot detection of Wnt3}

Frozen tissue samples and Wnt3-siRNA transfected cells were lysed by radioimmunoprecipitation assay buffer containing 1\% Nonidet P40 (NP-40), 0.5\% deoxycholate,
0.1\% sodium dodecyl sulfate (Beyotime, Haimen, Jiangsu, People's Republic of China) in the presence of protease inhibitor and centrifuged at $15,000 \times g$ for 10 minutes at $4^{\circ} \mathrm{C}$. The supernatant fraction was collected and the protein concentration was determined by the method of bicinchoninic acid assay (Beyotime). Equal amounts of protein extract ( $30 \mu \mathrm{g} /$ well) were separated by $10 \%$ sodium dodecyl sulfate-polyacrylamide gel electrophoresis. Separated proteins were electrotransferred to a $0.2 \mu \mathrm{m}$ polyvinylidene difluoride transfer membrane and blocked in 5\% nonfat milk in $1 \times$ Tris-buffered saline and Tween 20 for 1 hour. The blot was incubated with rabbit anti-Wnt3 at a dilution of 1:200 (Abcam) at $4^{\circ} \mathrm{C}$ overnight followed by horseradish peroxidase-conjugated secondary goat antirabbit antibody (GenePharma) at 1:2,000 dilution. Antibody against $\beta$-actin (GeneTex, Irvine, CA, USA) was used as loading control for the experiment. The membrane was then incubated with SuperSignal West Pico Chemiluminescent Substrates (Thermo Fisher Scientific) for 5 minutes and exposed to $\mathrm{X}$-ray films and developed in the dark room. The signal was quantified by densitometry using Gel-Pro Analyzer (Media Cybernetics, Rockville, MD, USA).

\section{Cell proliferation assay}

Cell proliferation was determined via cell counting kit-8 assay (Dojindo, Kumamoto, Japan). In brief, after transfected with siRNAs, cells in 96-well plate were added to $10 \mu \mathrm{L}$ of cell counting kit- 8 solution and incubated for a further 2 hours in $5 \% \mathrm{CO}_{2}$ at $37^{\circ} \mathrm{C}$. The absorbance was measured at $450 \mathrm{~nm}$ with an automated plate reader every 24 hours over 72 hours. The cellular growth curves were generated from the average values in triplicate wells for each group.

\section{Cell cycle and cell apoptosis analysis}

As the methods described earlier, MGC- 803 cells were transfected with different siRNAs for 48 hours. Subsequently, cells were digested with ethylenediaminetetraacetic acid-free trypsin and washed twice with cold PBS. For cell cycle assay, cells were resuspended in $500 \mu \mathrm{L}$ binding buffer with $5 \mu \mathrm{L}$ 7-amino-actinomycin and incubated at room temperature for 15 minutes. Prior to analysis, another $450 \mu \mathrm{L}$ binding buffer and $1 \mu \mathrm{L}$ annexin-V-phycoerythrin were added in sequence. Cells were suspended with $70 \%$ ethanol and kept at $20^{\circ} \mathrm{C}$ for 12 hours, then incubated with $0.25 \mathrm{mg} / \mathrm{mL}$ RNase A for 30 minutes at $37^{\circ} \mathrm{C}$ and $5 \mu \mathrm{L}$ propidium iodide at room temperature. After 30 minutes, cell cycle status was assessed with a BD Accuri C6 flow cytometer (BD, Franklin Lakes, NJ, USA) equipped with CellQuest Pro software (BD Biosciences, San Jose, CA, USA) according to the manufacturer's 
recommendations. To detect the apoptosis, cells were resuspended in $500 \mu \mathrm{L}$ binding buffer with $5 \mu \mathrm{L}$ 7-aminoactinomycin (7AAD) and incubated at room temperature for 15 minutes. Prior to analysis, another $450 \mu \mathrm{L}$ binding buffer and $1 \mu \mathrm{L}$ annexin-V-phycoerythrin were added in sequence. After 15 minutes, cell apoptosis was analyzed using flow cytometry and data analysis was performed by using Flow Jo software (Tree Star, Ashland, OR, USA).

\section{Transwell migration and invasion assays}

The effect of Wnt3-siRNA on the migration of MGC-803 cells was analyzed using a transwell migration system (Corning Incorporated, Corning, NY, USA). After transfection with siRNAs for 48 hours, MGC-803 cells were digested and disrupted into single cell suspensions, equal numbers of cells (1,000 cells) were plated into the upper transwell chambers in $2 \%$ FBS-containing medium and 15\% FBS-containing medium was added to the lower compartment of the chambers. The chambers with a pores sized of $8.0 \mu \mathrm{m}$ were incubated at $37^{\circ} \mathrm{C}$ in $5 \% \mathrm{CO}_{2}$ for 48 hours and cells remaining on the upper surface of the membrane were scratched by a cotton swab. The cells migrated through the pore and adhered to the lower surface were fixed with $4 \%$ paraformaldehyde for 20 minutes and stained with crystal violet $(0.05 \%)$ for another 30 minutes before photographed with the microscope.

\section{Wound healing migration assay}

MGC-803 cells were seeded into six-well plates and transfected with siRNAs for 48 hours, followed by scraped with a $100 \mu \mathrm{L}$ sterile pipette tip to generate two linear regions devoid of cells completely. The plates were then washed three times with PBS and refreshed with serum-free medium, cells were photographed and incubated in a $5 \% \mathrm{CO}_{2}$ at $37^{\circ} \mathrm{C}$. The cellular migrated distance was monitored and images were taken at 24 and 48 hours under microscope. The length and area of the wound region were measured using Image-Pro Plus 6.0 (Media Cybernetics) and the healing rate was calculated by the equation (width 1 - width 2)/width 1 , where width 1 and width 2 are the width values at 0 and 24/48 hours separately, and the width of wound was the ratio of area to length.

\section{Statistical analysis}

All statistical analyses were carried out using the SPSS version 11 software (SPSS Inc., Chicago, IL, USA). Oneway analysis of variance or two-sided Student's $t$-test was performed to evaluate statistical significance. Error bar for the experiments represents mean \pm standard deviation from three separate experiments. A $P$-value of $\leq 0.05$ was considered to be statistically significant.

\section{Results \\ Wnt3 is overexpressed in gastric cancer tissues}

Emerging evidence has revealed that canonical Wnt pathways play an important role in cellular proliferation and tumorigenesis. In order to determine whether Wnt signaling is aberrantly activated in tumor microenvironment, we examined the Wnt3 protein levels in 20 gastric carcinoma and 20 nonneoplastic gastric samples by immunohistochemical analysis. Wnt3 was found to be overexpressed in gastric cancer samples in contrast to the comparatively lower levels in normal tissues with a statistical significance $(P<0.05)$. Representative sections of Wnt3 expression in tumor tissues and normal ones are shown in Figure 1A and B. In a further effort to validate the earlier findings, we measured the expression of Wnt3 at protein levels in fresh gastric samples. As expected, immunoblot detection demonstrated a similar upregulation of Wnt3 protein level in gastric cancer tissues compared with nontumor ones (Figure 1C and D). Taken together, Wnt3 is upregulated in gastric carcinoma and may exert a carcinogenic effect in the occurrence and development of gastric tumors.

\section{Downregulation of $\mathrm{Wnt} 3$ in gastric cancer cells by Wnt3-siRNA}

To investigate the effect of Wnt3 on the survival of gastric cancer cells, we took advantage of the convenient siRNA technique to transfect the human gastric cancer cell lines, MGC-803 and SGC-7901. Four Wnt3-siRNA plasmids containing green fluorescent protein tag were used to target different Wnt3 transcript sequences. We first examined whether siRNAs could be transfected efficiently into the MGC-803 cells, as shown in Figure S1, after 24 hours of transfection, $>70 \%$ cells in all groups could be detected under fluorescent microscope compared with the light vision. To evaluate the specificity of siRNAs to the Wnt3 gene in MGC803 cells, we performed qRT-PCR and immunoblot analysis. We observed a $50 \%$ reduction of $W n t 3$ mRNA in the group of cells expressing Wnt3-714-siRNA or Wnt3-1188-siRNA compared with the control group and mock group (only treated with lipofectamine 2000), whereas Wnt3-337-siRNA and Wnt3-981-siRNA had no obvious interference effect on MGC-803 cells and were eliminated in the following experiments (Figure 2A). Immunoblot analysis showed a similar decrease in the $\sim 40 \mathrm{kDa} \mathrm{Wnt} 3$ protein levels compared with the control and mock groups (Figure 2B and C). The previous two Wnt3-siRNAs with more efficiently interfering capacity were selected for further investigation. Furthermore, we 


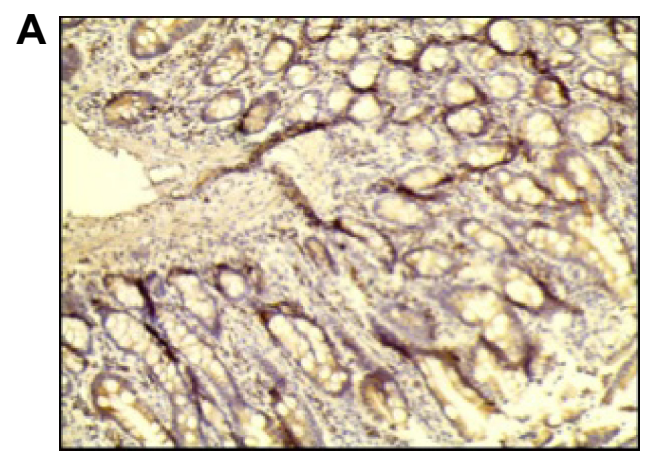

C Representative Wnt3 protein level in human gastric normal and cancer tissues

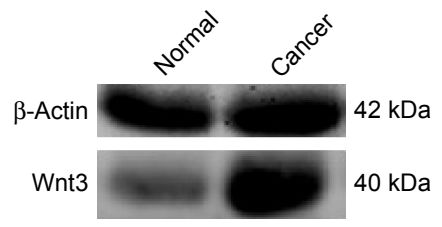

B
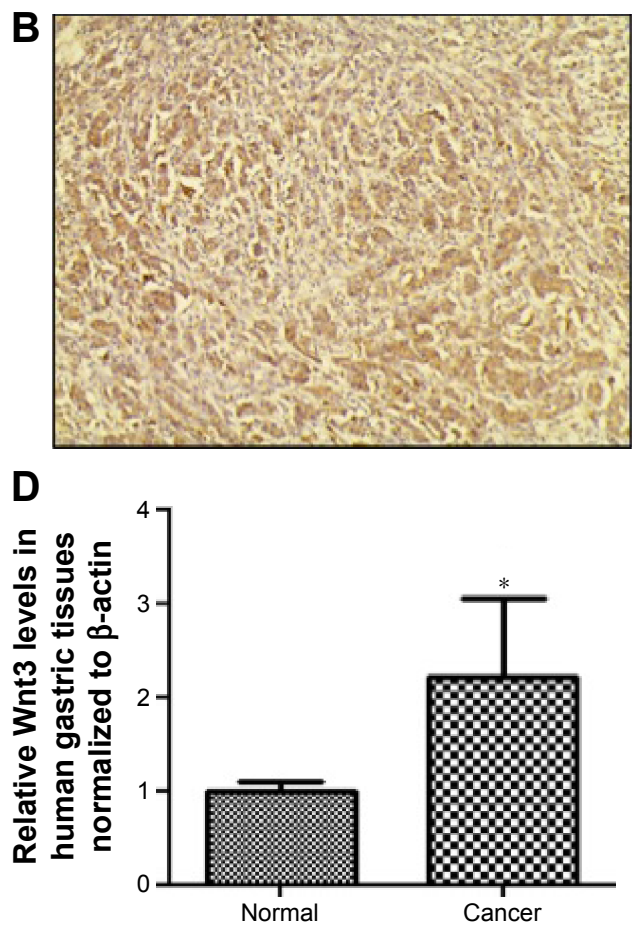

Figure I Expression of $\mathrm{Wnt} 3$ in human nonneoplastic gastric tissues and gastric cancer tissues.

Notes: Representative immunohistochemical staining of Wnt3 in human normal gastric tissues (A) or gastric cancer tissues (B). Magnification $\times 200$. (C and $\mathbf{D})$ Representative immunoblot of Wnt3 protein expression in human gastric cancer and corresponding nontumor tissues by Western blot analysis, normalized to human $\beta$-actin protein levels. Data represent the mean \pm standard deviation from ten groups of gastric tissues (normal and cancer). ${ }^{*} P<0.05$ compared with the corresponding adjacent normal gastric tissues.

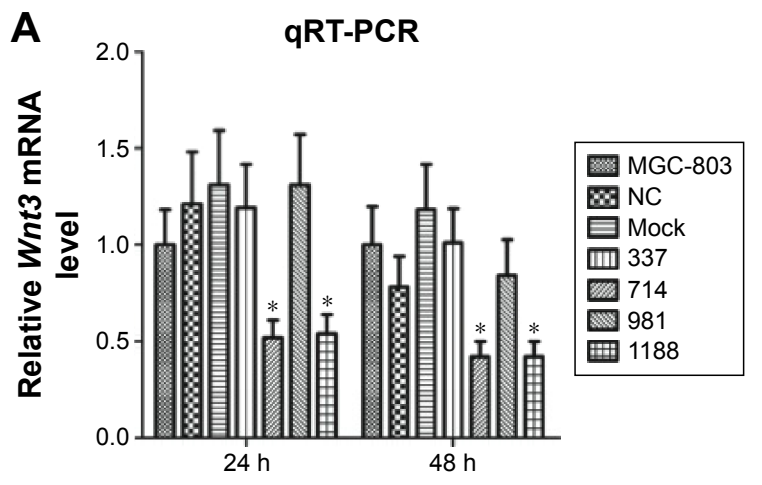

B
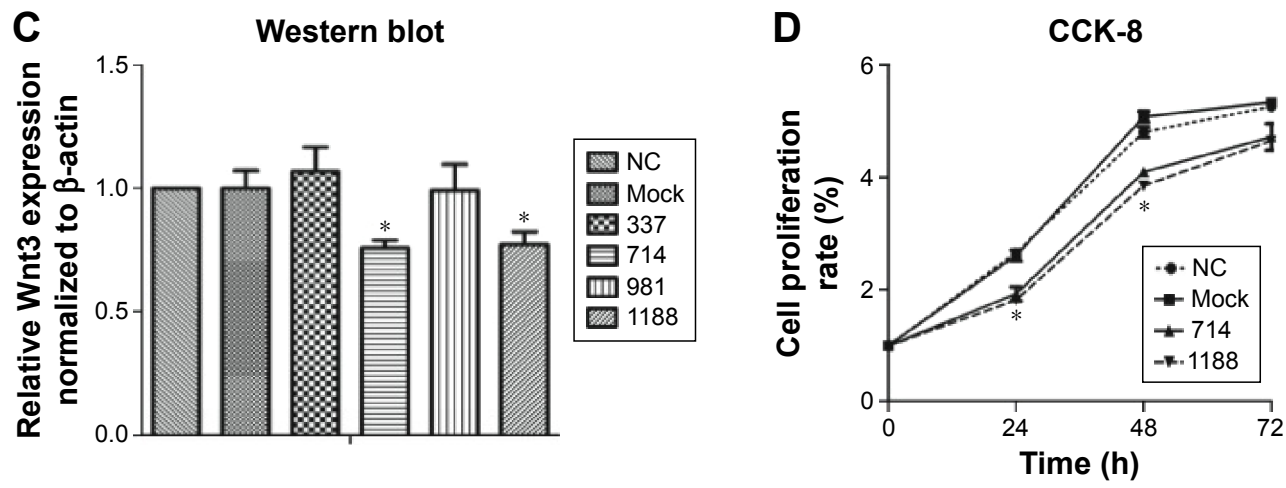

Figure 2 Wnt3 mRNA and protein expression levels were downregulated by Wnt3-siRNAs in MGC-803 cells.

Notes: (A) Representative qRT-PCR analysis of Wnt3 mRNA level in MGC-803 cells transfected with control, mock, or Wnt3-siRNAs targeting at sites of 337, 7I4, 98I, and I I88. $* P<0.05$ compared with the nontransfected MGC-803 cells. (B) Representative immunoblot showing Wnt3 protein levels extracts from MGC-803 cells transfected with control, mock, or above four Wnt3-siRNAs. (C) Densitometry was used to quantify relative Wnt3 protein levels normalized to $\beta$-actin shown in $B$; $* P<0.05$. (D) The proliferation rate of MGC-803 cells transfected with control, mock, Wnt3-7I4-siRNA, or Wnt3-I I88-siRNA over 72 hours. *P<0.05 compared with NC.

Abbreviations: CCK-8, cell counting kit-8; mRNA, messenger RNA; NC, control-siRNA group; qRT-PCR, quantitative real-time polymerase chain reaction; siRNAs, small interfering RNAs. 
found the gene expression of $\beta$-catenin and cyclin D1, two crucial components in Wnt/ $\beta$-catenin pathways, were inhibited when Wnt3 expression was silenced even though there was no change of the expression level of FZD7 (Figure S2), demonstrating that Wnt3 plays a key role in gastric tumorigenesis through activating the $\mathrm{Wnt} / \beta$-catenin pathways.

\section{Knockdown of Wnt3 inhibits proliferation} of gastric cancer cells

Enhanced cellular growth is one of the most important characteristics of tumor development. We next explored the impact of knockdown of Wnt3 on cellular growth viability of gastric cancer cells in vitro. MGC-803 cells were first transfected with Wnt3-714-siRNA, Wnt3-1188-siRNA, control siRNA or mock, respectively. The cell proliferation assay revealed that there was a significant inhibitory effect on cell proliferation in the two Wnt3-siRNA groups (Figure 2D). Microscopic analysis showed that the number of MGC-803 cell colonies formed in the two Wnt3-siRNA groups were significantly decreased as compared with the mock or control transfected ones (data not shown). A similar slower growth rate was also found in SGC-7901 cells transfected with Wnt3-1188-siRNA (Figure S3A). These results demonstrate that silencing Wnt3 could inhibit cell proliferation of gastric tumor cells.

\section{Silencing of Wnt3 expression arrests cell cycle and induces apoptosis in gastric cancer cells}

We proceeded to assess the effect of Wnt3 depletion on cell cycle and apoptosis of MGC-803 cells. The flow cytometry was performed to assay the cell cycle distribution of MGC803 cells at 48 hours after transfection with mock, control, or Wnt3-targeted siRNAs. The results demonstrated that silencing of Wnt3 inhibited cell cycle progression through arresting $\mathrm{S} / \mathrm{G} 2$ transition, as shown in Figure 3, the proportion of cells in G0/G1 phase (Wnt3-714-siRNA for 32.65\% and Wnt3-1188siRNA for $27.03 \%$ ) after transfection with Wnt3-siRNAs was obviously lower than that in the mock or control cells $(42.47 \%$ and $44.4 \%$ ), while on the contrary, a much high percentage of
A

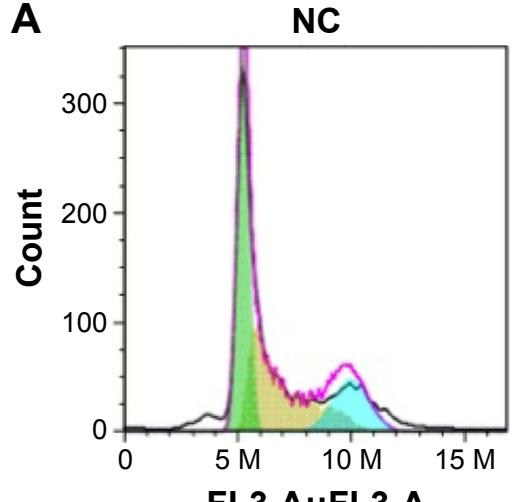

FL3-A::FL3-A
B

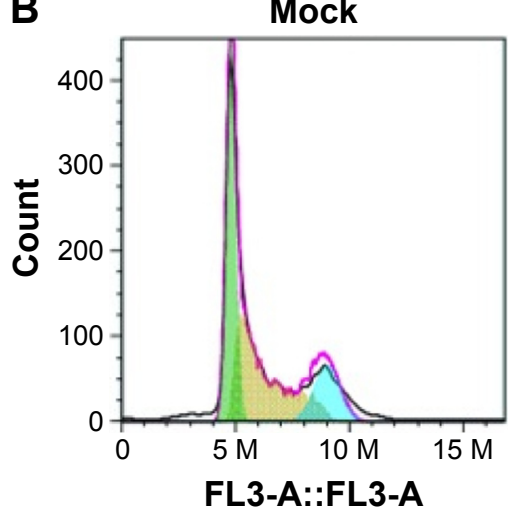

C

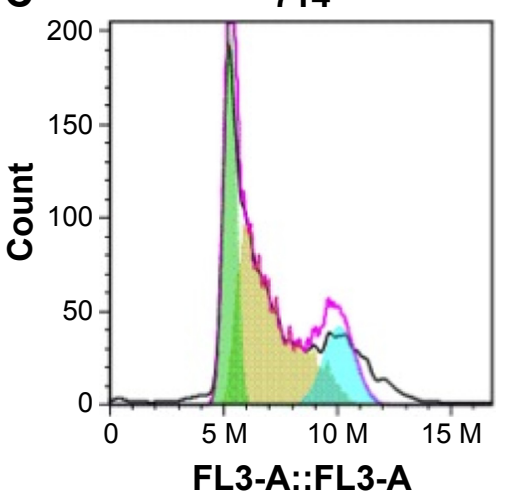

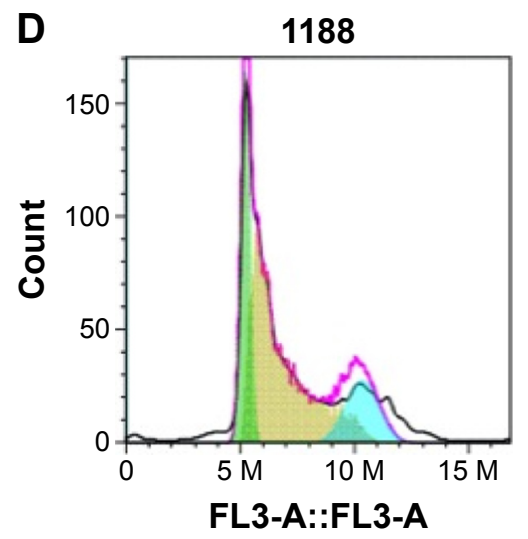

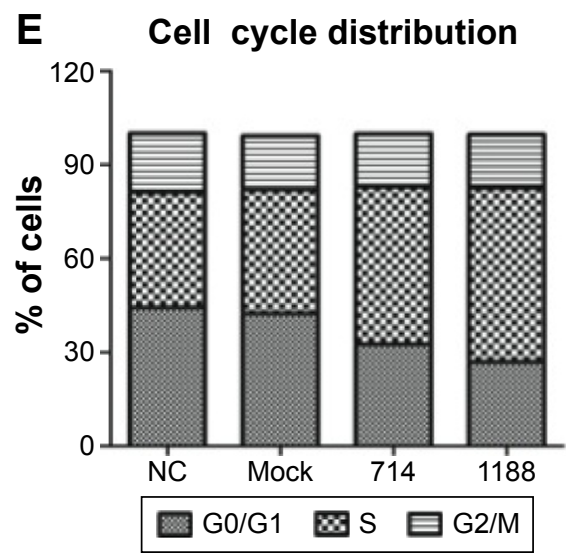

Figure 3 Effect of knockdown Wnt3 on the cell cycles of gastric cells.

Notes: (A-D) Flow cytometry analysis of cell cycle distribution in MGC-803 after transfected with control, mock, Wnt3-7I4-siRNA, or Wnt3-I I88-siRNA for 48 hours. (E): The percentages of cells in the G0/GI, S, G2/M phases of the cell cycle in each group were measured by using the CellQuest Pro software. The values in each group represent the average values from three samples. FL3-A indicates that the fluorescence signal was detected by FL3 channel in FACS analysis.

Abbreviations: FACS, fluorescence-activated cell sorting; NC, control-siRNA group; siRNA, small interfering RNA. 


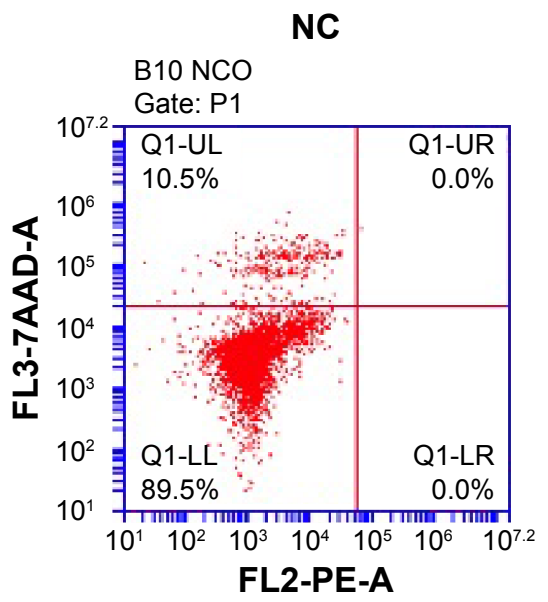

1188

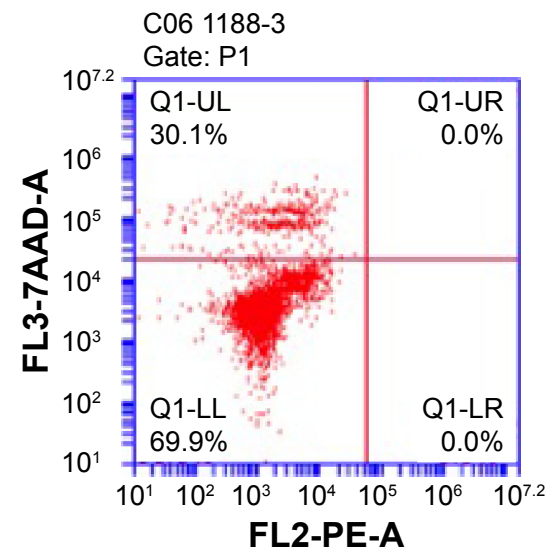

Mock

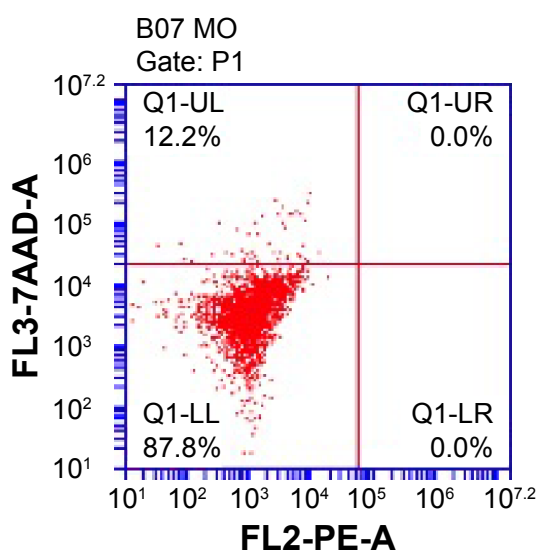

714

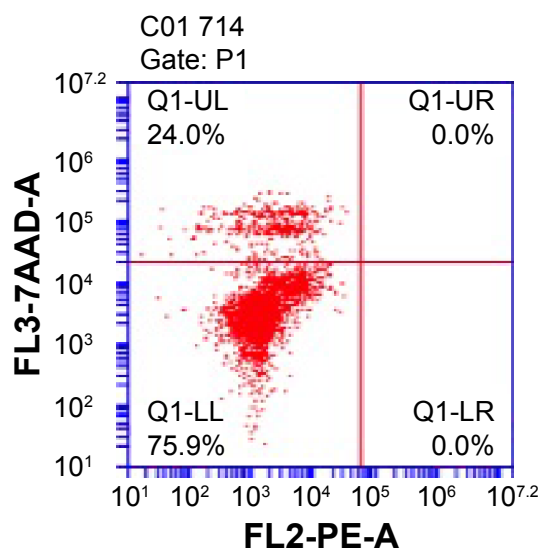

Apoptosis detection

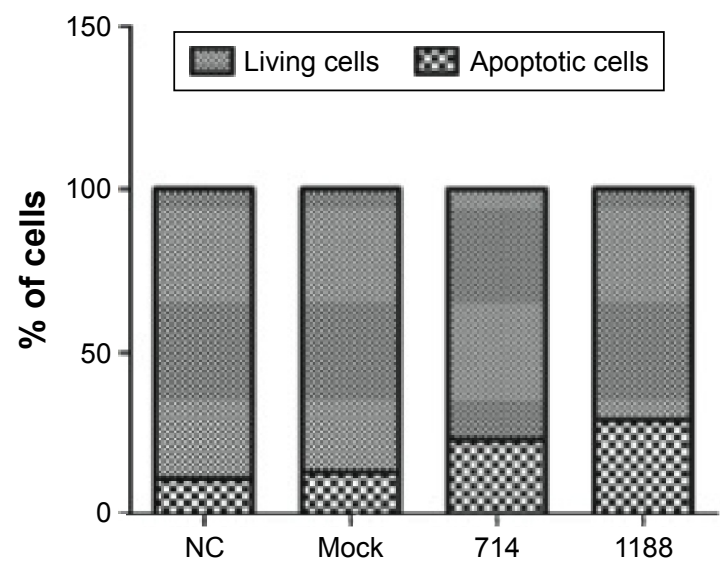

Figure 4 Downregulation of Wnt3 by siRNA induces the apoptosis of gastric cells.

Notes: Flow cytometry apoptosis detection of MGC-803 cells after transfected with control, mock, Wnt3-7I4-siRNA, or Wnt3-II 88-siRNA for 48 hours, cells were double stained with annexin $\mathrm{V}$ and 7AAD. Lower right: The percentages of living or apoptotic cells in each group were analyzed by FlowJo software (Tree Star, Ashland, OR, USA). The values in every group represent the average from three samples. FL2 and FL3 indicates channels used in FACS analysis.

Abbreviations: 7AAD, 7-amino-actinomycin D; B07 MO, mock; BI0 NCO, control; C0I 7I4, Wnt3-7I4-siRNA transfected; C06 I888-3, Wnt3-I888-siRNA transfected; FACS, fluorescence-activated cell sorting; LL, lower-left; LR, lower-right; NC, control-siRNA group; PI, parameter number I; PE-A, annexin V positive; QI, quadrant label; siRNA, small interfering RNA; UL, upper-left; UR, upper-right.

cells in the S phase was found in Wnt3 knockdown MGC-803 cells (Wnt3-714-siRNA for 50.42\% and Wnt3-1188-siRNA for $55.73 \%$ ) than that in the mock or control transfected ones (39.69\% and 36.92\%). Next, we investigated the impact of Wnt3 depletion on the cell apoptosis of MGC-803 cells using annexin V/7-amino-actinomycin D staining, our results illustrated that silencing of Wnt3 increased number of apoptotic cells relative to the mock or control group (Figure 4). Taken together, these results revealed that downregulation of Wnt3 expression arrests cell cycle and promotes apoptosis in MGC-803 cells.

\section{Knockdown of Wnt3 decreases migration and invasion of gastric cancer cells}

Cell migration and invasion are important properties of tumor metastasis. To determine the effects of Wnt 3 on gastric cancer cell migration and invasion, we conducted the invasion assay by using a transwell migration system and wound healing assay through a scratch method. In the transwell assay, silencing Wnt3 confers decreased penetration capacity of MGC-803 gastric cells via the matrigel-coated membrane, in contrast to the control-siRNA and mock transfected cells (Figure 5A and B). The results were confirmed using SGC7901 gastric cancer cells (Figure S3B). Furthermore, wound healing assay demonstrated that Wnt 3 knockdown decreased the migration capability of MGC-803 cells approximately 1.8 times than that of the control or mock transfected ones (Figure 5C and D). These results suggest that downregulation of Wnt3 reduced the migration and invasion ability of gastric cancer cells in vitro.

\section{Discussion}

Gastric carcinoma is a chronic, complex, and multifactorial disease that results from the interaction among genetic and environmental risk factors at the level of stomach mucosa. Excessive intake of salty or pickled food, smoking, and 
A

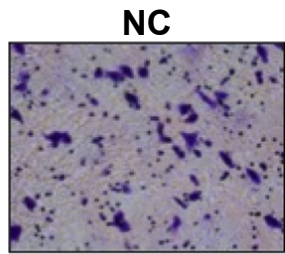

714

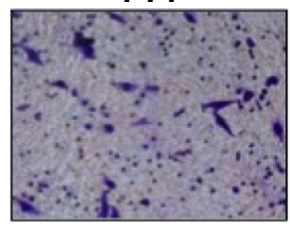

NC

C
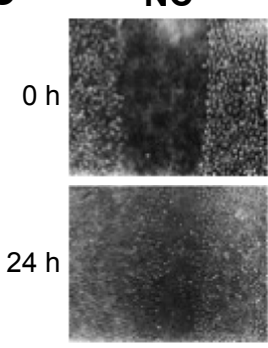

$48 \mathrm{~h}(x-2)$
Mock
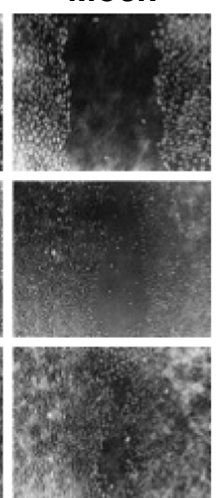

Mock

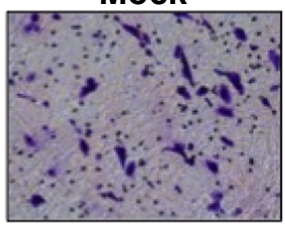

1188

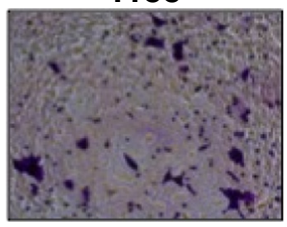

714
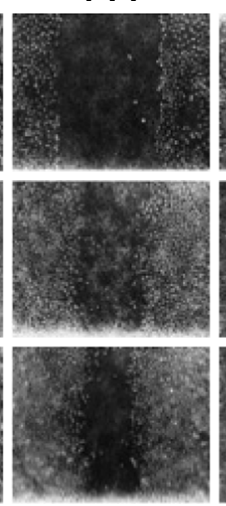

1188
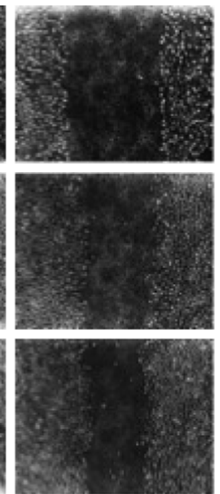

B

Transwell migration assay

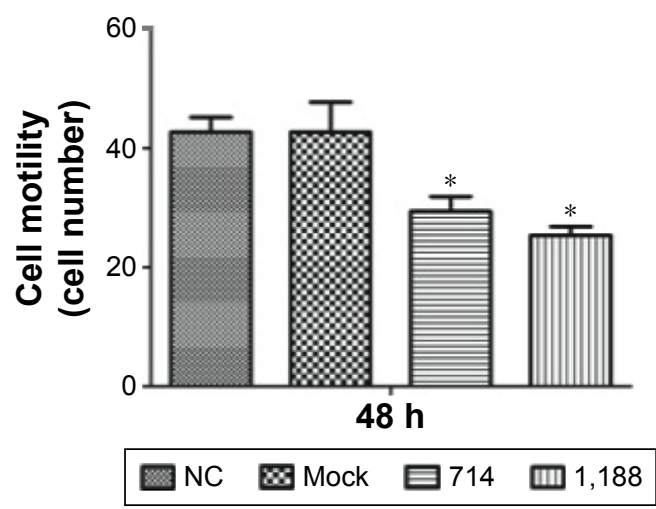

Wound healing assay

D

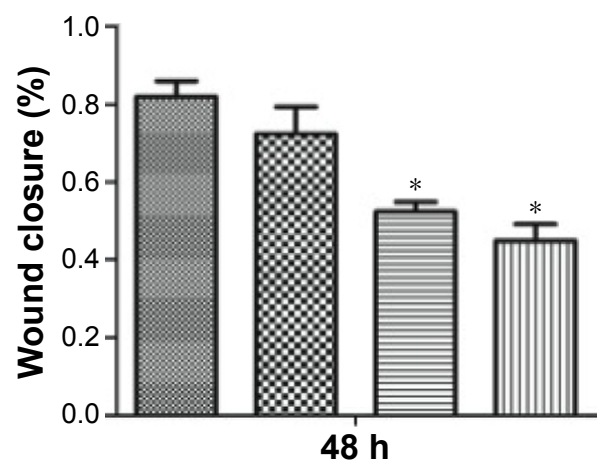

Figure 5 Silencing Wnt3 inhibits the invasion and migration of gastric cells.

Notes: (A) Representative photographs of stained MGC-803 cells attached to the bottom of matrigel-coated membrane (magnification $\times 100$ ), cells were transfected with control, mock, Wnt3-7I4-siRNA, or Wnt3-II88-siRNA for 48 hours separately. (B) Both Wnt3-7I4-siRNA and Wnt3-II88-siRNA suppressed MGC-803 cell invasion in comparison to NC; $* P<0.05$. (C) Representative photographs of MGC-803 cells taken at $0,24,48$ hours postwound (magnification $\times 40$ ) after treated with control, mock, Wnt3-7/4-siRNA, or Wnt3-I I88-siRNA. (D) Quantification of the wound healing rate at 48 hours postwound by measuring the remaining unhealed area using Image-Pro Plus 6.0 (Media Cybernetics, Rockville, MD, USA). $* P<0.05$ compared with NC.

Abbreviations: NC, control-siRNA group; siRNA, small interfering RNA.

H. pylori infection are thought to be important factors in malignant transformation of gastric mucosal cells and gastric carcinogenesis. The most effective used means for the early detection of gastric cancer is the endoscopy, with a sensitivity ranges from $77 \%$ to $84 \% .{ }^{12}$ However, relying heavily on operating skills of the endoscopic physicians and higher medical costs limits its convenient application. Serum pepsinogen (PG) test based on the level of PG I and PG I/II ratio is another effective method for the early detection of gastric cancer, especially in combination with barium swallow X-ray examination. ${ }^{13}$ Other functional biomarkers investigated in gastric cancer contain various metabolites of four major biomolecules, ${ }^{14}$ protein biomarkers such as MG7-Ag, Fas, FasL, Reg IV, other molecules in serum such as CEA, CA19-9, CA72-4, and IPO-38, ${ }^{15}$ microRNAs including miR-185, miR-20a, miR-210, miR-25, and miR-92b. ${ }^{16}$ Liang et al ${ }^{17}$ recently found that transforming growth factor- $\beta 1$-(p-Smad3)-RBP2-E-cadherin-Smad3 feedback circuit may be a novel potential mechanism for malignant progression and metastasis of gastric cancer and inhibition of RBP2 expression may be a therapeutic option to fight gastric cancer.

Previous studies have demonstrated that deregulation of Wnt signaling pathway is found in $\sim 30 \%-50 \%$ of patients diagnosed with gastric cancer and closely associated with a variety of human diseases, including hereditary diseases such as amelia and familiar adenomatous polyposis; ${ }^{18}$ several types of other human cancers such as prostate cancer, breast cancer, colorectal cancer, and melanoma; ${ }^{11}$ Parkinson's disease as well as metabolic diseases including obesity and type II diabetes. ${ }^{19,20}$ These findings imply a crucial role for the Wnt pathway in physiological embryonic development and tissue homeostasis. However, questions about the expression pattern of Wnt 3 and complete knowledge of its activation in gastric cancer are not well understood yet. In this study, we elucidated the biological significance of Wnt3 in human gastric cancer. It is interesting to find that expression of Wnt3 protein is increased 
in gastric cancer from immunohistochemical analyses and patient fresh samples compared with control groups; similar results were also observed in gastric cancer for other Wnt family members including Wnt1, Wnt2/2b, Wnt5a, Wnt6, and Wnt10a. ${ }^{21}$

Wnt signaling pathways are canonical signal transduction cascades, which play a major role during embryonic development, cellular proliferation, survival, and apoptosis. Wnt 3 is one of the fundamental components that recognize and bind the receptor complex to initiate the activation of the pathways. In the status of tumor microenvironment, activation of Wnt pathway by unknown reasons led to the accumulation of $\beta$-catenin in cytoplasm. Once entering the nucleus, it engages DNA-bound T-cell factor transcription factors as a transcriptional coregulator and activates Wnt target gene expression such as AXIN2, SMAD7, CCND1/ cyclin $D$, and $M Y C$ to induce the malignant transformation. ${ }^{22}$ In nontransformed hepatic cells, stable transfection of Wnt 3 and FZD7 activates the Wnt $3 / \beta$-catenin pathway and promotes the acquisition of a malignant phenotype. ${ }^{9}$ In trastuzumab-resistant HER2-overrepressing breast cancer cells, expression of Wnt3 also activates this cascade and promotes a partial epithelial-to-mesenchymal transition. ${ }^{23}$ The overexpression of Wnt3 presented here concurred with those published observations and strongly verified that Wnt3 has a crucial function in gastric carcinogenesis and may serve as novel therapeutic target of gastric cancer. However, limited to the fresh gastric cancer samples and medical records, we had no capability to determine the correlation of Wnt3 levels with the clinical staging, pathologic grading, and metastasis of gastric cancer. Future research based on an enlarged number of tissues can elucidate whether Wnt3 transcript levels as well as protein are associated with different clinic pathological features in gastric cancer.

The occurrence and progression of malignant tumors are mainly attributed to the imbalance of cellular proliferation and apoptosis. In the present study, we addressed the question whether the aberrant expression of Wnt 3 destroyed this dynamic balance. In order to explore the molecular mechanism, we knocked down Wnt3 using siRNAs in gastric cancer cells. Indeed, our results demonstrated that interfering against Wnt3 mRNA could downregulate its effector molecules including $\beta$-catenin and cyclin D1 in Wnt signaling pathway, inhibit cellular proliferation, halt cell cycle in S/G2 phase, suppress migration and invasion abilities of cells, whereas induce cell apoptosis. In line with this finding, studies in breast cancer cells showed that depleting Wnt3 displayed reduced invasiveness and restored growth-inhibitory effects of trastuzumab, which are assumed to be the restored cytoplasmic expression of $\beta$-catenin and decreased expression of EGF receptor. ${ }^{23}$ Consistently, knocking down Wnt3 by siRNA in KMS-5 myeloma cells overcame cell adhesion-mediated drug resistance of myeloma cells. ${ }^{24}$ In addition, one recent study has reported that Wnt3 ligand augmented the $\beta$-catenin activity of MCF7 cells in a Dickkopf-1-insensitive manner and anti-LRP5 antibody attenuated the activity of Wnt $3 / \beta$-catenin, inhibited cell proliferation, and induced apoptosis in LRP5Delta-positive MCF7 and T-47D breast cancer cells. ${ }^{25}$ These findings further validated the essential oncogenic function of Wnt3 in the pathogenesis of various human cancers. Therefore, downregulation of Wnt3 could be a promising strategy for the treatment of cancers, even though it remains obscure to what extent an antitumorigenic role of Wnt3 may contribute to the prevention of cancers.

\section{Conclusion}

The earlier findings support the idea that upregulation of Wnt3 is a crucial step in the tumorigenesis of gastric cancer. Downregulation or suppression of Wnt3 in gastric cancer cells downregulated the expression of $\beta$-catenin and cyclin D1 in Wnt3/ $\beta$-catenin pathway, halted cancer cell proliferation and migration, induced apoptosis, hence targeting Wnt3 by siRNA technology may be one of the convenient and effective ways for cancer treatment. Further analysis is required to elucidate the changes of more downstream effectors through which Wnt3 promotes the tumorigenicity in gastric cancer. Taken together, it is intriguing to demonstrate that Wnt 3 could be a novel therapeutic target for treatment of gastric cancer, and developing new drugs based on targeting Wnt3 may be a potential direction for antigastric cancer therapy.

\section{Acknowledgments}

This work is supported by the National Natural Science Foundation of China (Grant number 81370537) and the Fundamental Research Funds for the Central Universities (Grant number YS1407 and Buctrc201305) to Y-DW, the National Natural Science Foundation of China (Grant number 81270522 and Grant number 81472232), Program for Science and Technology Innovation Talents in Universities of Henan Province (HASTIT, Grant number 13HASTIT024), and Plan for Scientific Innovation Talent of Henan Province to W-DC, Key Natural Science Fund of Education Department of Inner Mongolia to H-SW (NJZZ12149).

\section{Disclosure}

The authors report no conflicts of interest in this work. 


\section{References}

1. Bernard WS, Christopher PW, Chris W. "Chapter 1.1". World Cancer Report 2014. Geneva: World Health Organization; 2014.

2. Graham DY. Helicobacter pylori update: gastric cancer, reliable therapy, and possible benefits. Gastroenterology. 2015;148(4):e719-731.e713.

3. Orditura M, Galizia G, Sforza V, et al. Treatment of gastric cancer. World J Gastroenterol. 2014;20(7):1635-1649.

4. Baker NE. Transcription of the segment-polarity gene wingless in the imaginal discs of Drosophila, and the phenotype of a pupal-lethal wg mutation. Development. 1988;102(3):489-497.

5. Klaus A, Birchmeier W. Wnt signalling and its impact on development and cancer. Nat Rev Cancer. 2008;8(5):387-398.

6. Gao C, Chen YG. Dishevelled: The hub of Wnt signaling. Cell Signal. 2010;22(5):717-727.

7. Anastas JN, Moon RT. WNT signalling pathways as therapeutic targets in cancer. Nat Rev Cancer. 2013;13(1):11-26.

8. Kahn M. Can we safely target the WNT pathway? Nat Rev Drug Discov. 2014;13(7):513-532.

9. Nambotin SB, Tomimaru Y, Merle P, Wands JR, Kim M. Functional consequences of WNT3/Frizzled7-mediated signaling in non-transformed hepatic cells. Oncogenesis. 2012;1:e31.

10. Kato S, Hayakawa Y, Sakurai H, Saiki I, Yokoyama S. Mesenchymaltransitioned cancer cells instigate the invasion of epithelial cancer cells through secretion of WNT3 and WNT5B. Cancer Sci. 2014;105(3): 281-289.

11. Voloshanenko O, Erdmann G, Dubash TD, et al. Wnt secretion is required to maintain high levels of Wnt activity in colon cancer cells. Nat Commun. 2013;4:2610.

12. Hamashima C, Shibuya D, Yamazaki H, et al. The Japanese guidelines for gastric cancer screening. Jpn J Clin Oncol. 2008;38(4):259-267.

13. Ohata H, Oka M, Yanaoka K, et al. Gastric cancer screening of a highrisk population in Japan using serum pepsinogen and barium digital radiography. Cancer Sci. 2005;96(10):713-720.

14. Chan AW, Gill RS, Schiller D, Sawyer MB. Potential role of metabolomics in diagnosis and surveillance of gastric cancer. World $J$ Gastroenterol. 2014;20(36):12874-12882.
15. Hao Y, Yu Y, Wang L, et al. IPO-38 is identified as a novel serum biomarker of gastric cancer based on clinical proteomics technology. J Proteome Res. 2008;7(9):3668-3677.

16. Zhou X, Zhu W, Li H, et al. Diagnostic value of a plasma microRNA signature in gastric cancer: a microRNA expression analysis. Sci Rep. 2015;5:11251.

17. Liang X, Zeng J, Wang L, et al. Histone demethylase RBP2 promotes malignant progression of gastric cancer through TGF-beta1(p-Smad3)-RBP2-E-cadherin-Smad3 feedback circuit. Oncotarget. 2015;6(19):17661-17674.

18. Kinzler KW, Nilbert MC, Su LK, et al. Identification of FAP locus genes from chromosome 5q21. Science. 1991;253(5020):661-665.

19. Isakson $P$, Hammarstedt $A$, Gustafson $B$, Smith U. Impaired preadipocyte differentiation in human abdominal obesity: role of Wnt, tumor necrosis factor-alpha, and inflammation. Diabetes. 2009;58(7):1550-1557.

20. Shruster A, Offen D. Targeting neurogenesis ameliorates danger assessment in a mouse model of Alzheimer's disease. Behav Brain Res. 2014;261:193-201.

21. Chiurillo MA. Role of the Wnt/beta-catenin pathway in gastric cancer: An in-depth literature review. World J Exp Med. 2015;5(2):84-102.

22. Behrens J, von Kries JP, Kuhl M, et al. Functional interaction of betacatenin with the transcription factor LEF-1. Nature. 1996;382(6592): 638-642.

23. Wu Y, Ginther C, Kim J, et al. Expression of Wnt3 activates Wnt/betacatenin pathway and promotes EMT-like phenotype in trastuzumabresistant HER2-overexpressing breast cancer cells. Mol Cancer Res. 2012; 10(12):1597-1606.

24. Kobune M, Chiba H, Kato J, et al. Wnt3/RhoA/ROCK signaling pathway is involved in adhesion-mediated drug resistance of multiple myeloma in an autocrine mechanism. Mol Cancer Ther. 2007;6(6):1774-1784.

25. Bjorklund P, Svedlund J, Olsson AK, Akerstrom G, Westin G. The internally truncated LRP5 receptor presents a therapeutic target in breast cancer. PLoS One. 2009;4(1):e4243. 


\section{Supplementary materials}

$24 \mathrm{~h}$
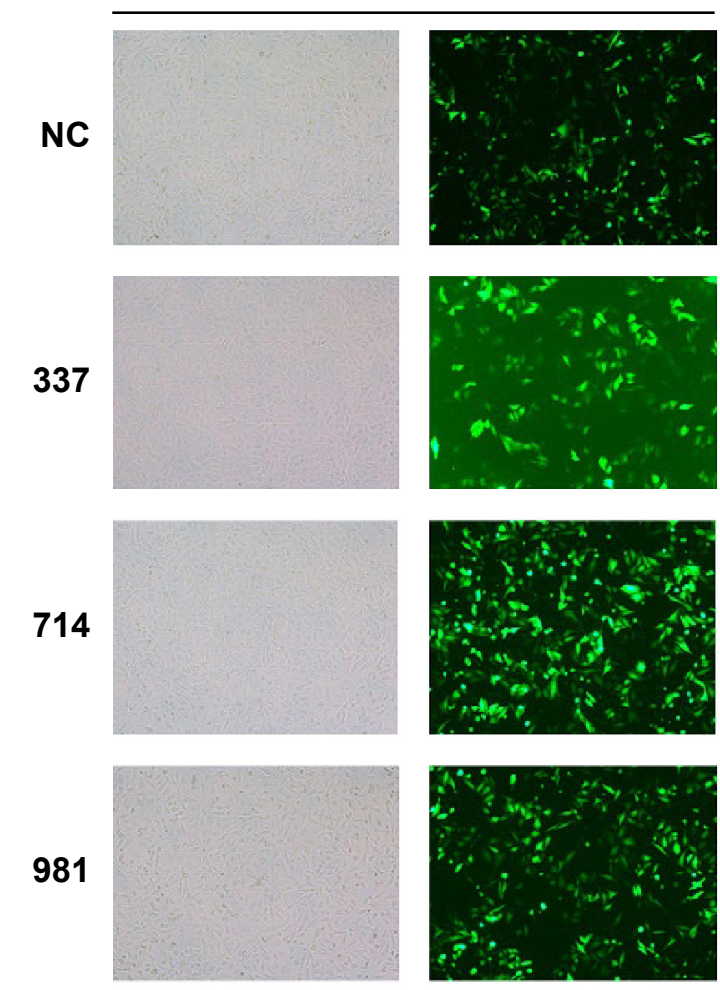

1188
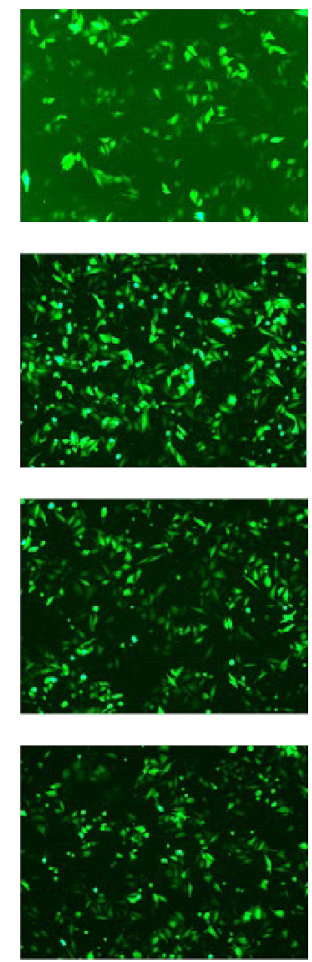

$48 \mathrm{~h}$
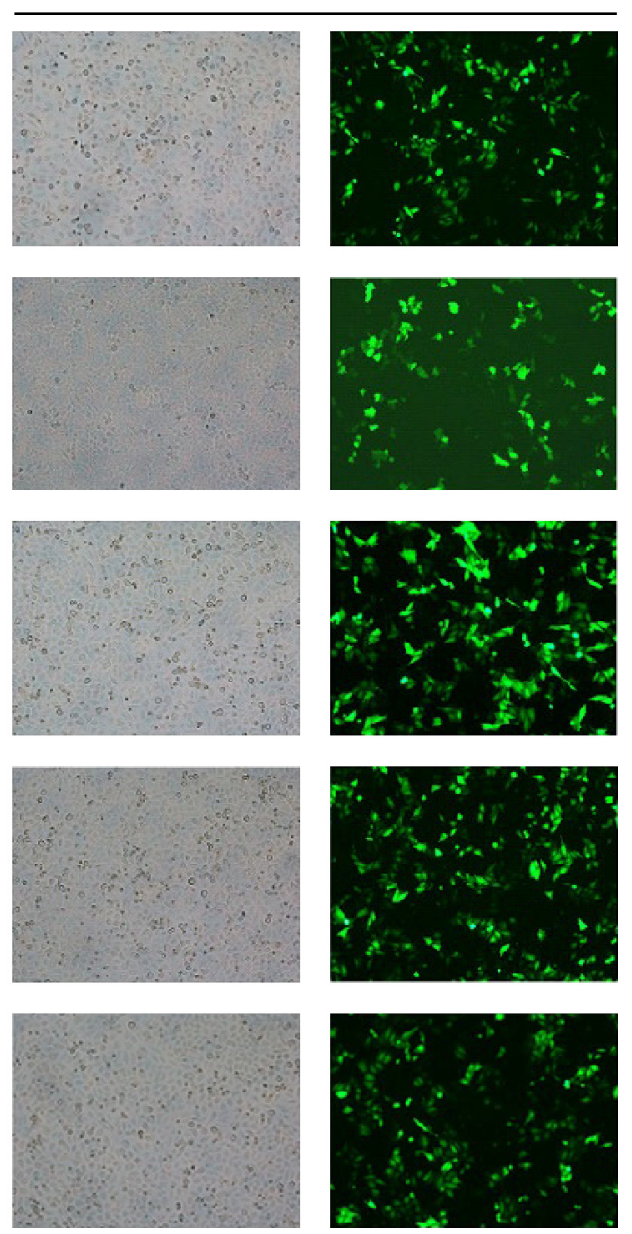

Figure SI Expression efficiencies of Wnt3-siRNAs in MGC-803 cells.

Notes: Representative photographs of MGC-803 cells taken at 24 or 48 hours under light vision or fluorescent microscope (magnification $\times 40$ ), after transfected with control, mock, Wnt3-337-siRNA, Wnt3-714-siRNA, Wnt3-981 siRNA, or Wnt3-1 188-siRNA.

Abbreviations: NC, control-siRNA group; siRNA, small interfering RNA.

A

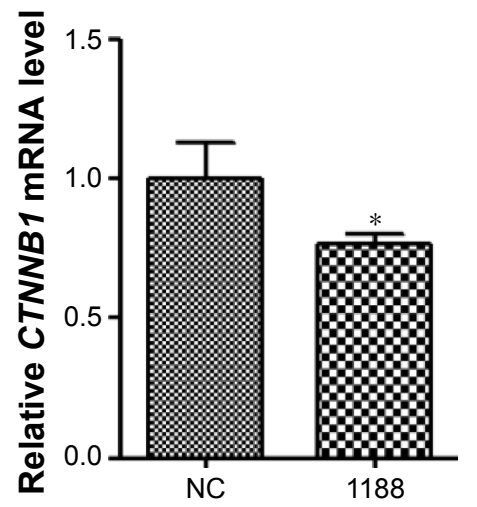

B

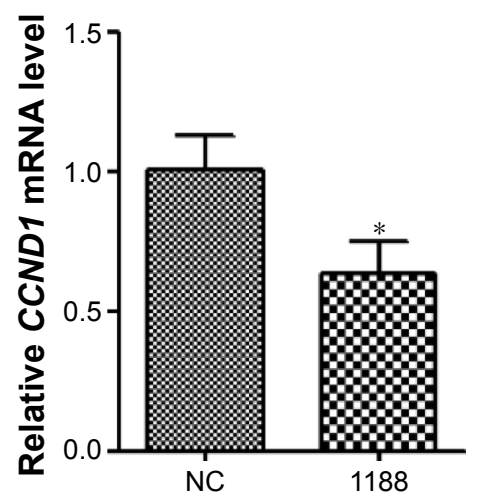

C

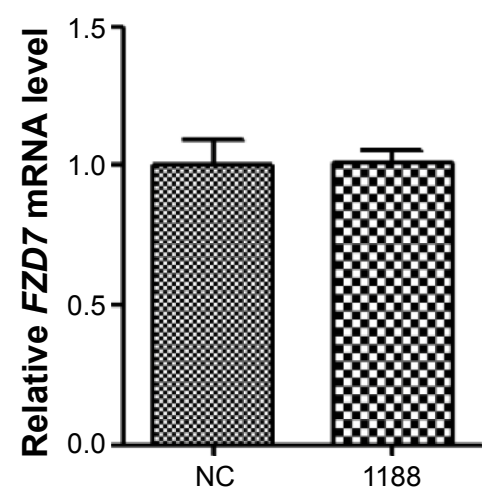

Figure S2 The mRNA levels of Wnt3 effector molecules were downregulated by Wnt3-siRNA in MGC-803 cells.

Notes: (A) qRT-PCR analysis of CTNNBI ( $\beta$-catenin) mRNA levels in MGC-803 cells transfected with control or Wnt3-siRNAs targeting at sites of I I88. $* P<0.05$ compared with NC. (B) The mRNA levels of CCNDI (cyclin DI) in MGC-803 cells transfected with control or Wnt3-II88-siRNA. *P<0.05 compared with the NC group. (C) The mRNA levels of FZD7 (frizzled class receptor 7) in MGC-803 cells transfected with control or Wnt3-I I88-siRNA.

Abbreviations: NC, control-siRNA group; qRT-PCR, quantitative real time polymerase chain reaction; siRNA, small interfering RNA. 
A

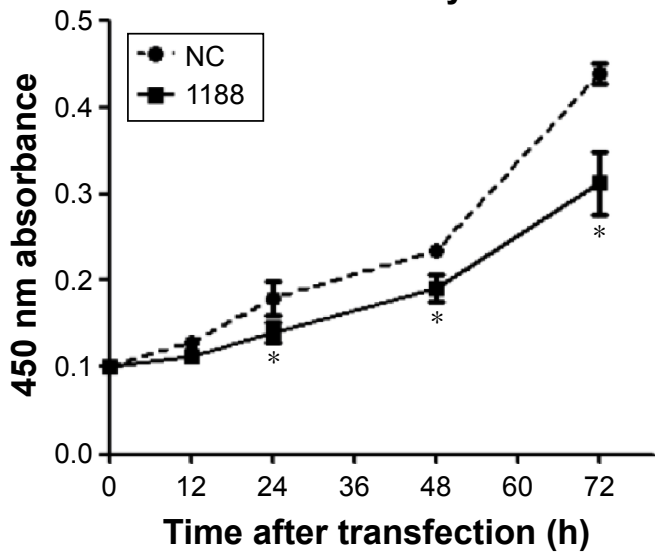

B

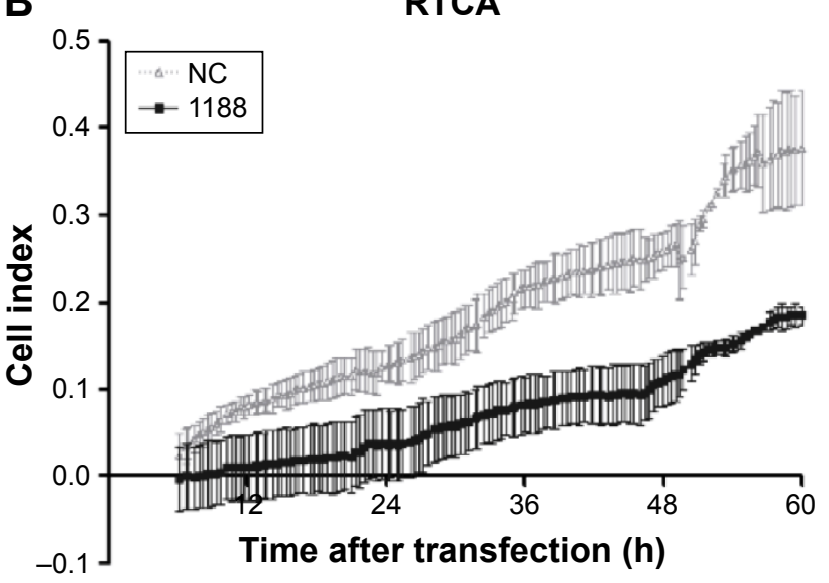

Figure S3 Silencing Wnt3 suppressed the proliferation and migration of SGC-790 I cells.

Notes: The SGC-790 I cells were transfected with control or Wnt3-I I88-siRNA. (A) MTT assays revealed knockdown of Wnt3 significantly inhibited proliferation of SGC790 I cells, $* P<0.05$. (B) RTCA showed the cell migration ability was obviously suppressed in SGC-790I cells transfected with Wnt3-I I88-siRNA. $* P<0.05$ compared with the NC group. The migration index was recorded every 15 minutes from 6 to 60 hours posttransfection using RTCA software.

Abbreviations: MTT, (3-(4, 5-dimethylthiazolyl-2)-2, 5-diphenyltetrazolium bromide; NC, control-siRNA group; RTCA, real-time cell analysis; siRNA, small interfering RNA.

\section{Publish your work in this journal}

OncoTargets and Therapy is an international, peer-reviewed, open access journal focusing on the pathological basis of all cancers, potential targets for therapy and treatment protocols employed to improve the management of cancer patients. The journal also focuses on the impact of management programs and new therapeutic agents and protocols on patient perspectives such as quality of life, adherence and satisfaction. The manuscript management system is completely online and includes a very quick and fair peer-review system, which is all easy to use. Visit http://www.dovepress.com/testimonials.php to read real quotes from published authors. 